(2) Open Access Full Text Article

\title{
Promising Swellable Floating Bupropion Tablets: Formulation, in vitro/in vivo Evaluation and Comparative Pharmacokinetic Study in Human Volunteers
}

This article was published in the following Dove Press journal:

Drug Design, Development and Therapy

\author{
Mahmoud Teaima (D) \\ Magdi M. Abdel Hamid $\mathbb{D}^{2}$ \\ Nabil A. Shoman ${ }^{2}$ \\ Bhaskara R. Jasti ${ }^{3}$ \\ Mohamed A. El-Nabarawi ${ }^{1}$ \\ 'Department of Pharmaceutics and \\ Industrial Pharmacy, Faculty of Pharmacy, \\ Cairo University, Cairo, Egypt; \\ ${ }^{2}$ Department of Pharmaceutics and \\ Industrial Pharmacy, Faculty of Pharmacy, \\ Ahram Canadian University, Giza, Egypt; \\ ${ }^{3}$ Department of Pharmaceutics and \\ Medicinal Chemistry, Thomas J. Long \\ School of Pharmacy \& Health Sciences, \\ University of the Pacific, Stockton, \\ California, USA
}

\begin{abstract}
Purpose: Bupropion is an antidepressant drug that facilitates weight loss. It is a highly water-soluble drug that needs multiple dosing, so it is considered a potential candidate for oral controlled-release dosage form. The aim of this research was to formulate and evaluate satiety-inducing swellable floating bupropion tablets by direct compression targeting depression associated with eating disorders. Various combinations of natural and semi-synthetic hydrogels were selected to achieve maximum swelling and remaining buoyant in the stomach. This synergistically enhances weight loss by increasing satiety.
\end{abstract}

Methods: An I-optimal mixture design was conducted to establish the optimal quantitative composition of tablets. Friability, floating lag time, swelling index after 4 and 8 hours, along with the percent of bupropion released at 1 and 8 hours were selected as dependent variables. The optimized formulation was characterized by physicochemical properties, thermal stability, and chemical interaction. In vivo radiographic evaluation of gastric residence besides, the oral bioavailability relative to marketed Wellbutrin ${ }^{\circledR}$ sustained-release tablets were investigated using human volunteers.

Results: The optimized formulation $(73.3 \mathrm{mg}$ xanthan, $120 \mathrm{mg}$ glucomannan, $8.4 \mathrm{mg}$ tamarind kernel powder, $78.3 \mathrm{mg}$ HPMC K15M) was achieved with the overall desirability equals 0.782 . In vivo radiographic study showed that formulation was retained for $>8$ hours in the stomach. Compared with the marketed BUP tablets, the $\mathrm{C}_{\max }$ was almost the same with a significant increase $(\mathrm{p}=0.004)$ for $\mathrm{T}_{\max }$.

Conclusion: Using combinations of these hydrogels would be promising gastroretentive delivery systems in the control of bupropion rate release with enhanced floating and swelling features.

Keywords: gastroretentive drug delivery, natural hydrogels, HPMC, depression, eating disorder

\section{Introduction}

Bupropion (BUP) is a norepinephrine and dopamine reuptake inhibitor that marketed for depression treatment. Multiple research revealed that bupropion is able to improve hyperphagic-depressed individuals and overweight or obese patients with depression. ${ }^{1}$ Although BUP required several daily dosings for therapy, besides its inconvenience of $\mathrm{C}_{\max }$-related adverse effects that are dose-dependent. Thus, a persistent challenge needed for formulating a controlled-release delivery system
Correspondence: Mahmoud Teaima Department of Pharmaceutics and Industrial Pharmacy, Faculty of Pharmacy, Cairo University, Kasr El-Aini Street, Cairo II562, Egypt

Email mahmoud.teaima@pharma.cu.edu.eg 
to improve compliance and tolerability. Oral controlledrelease delivery systems aim to significantly prolong the gastric residence time of dosage forms in the region of the stomach to release drug at the desired rate. ${ }^{2}$

Gastroretentive drug delivery system is one such example that could be retained in the stomach for a long time to release the drug in a sustained manner at the absorption site. ${ }^{3}$ Floating drug delivery system is considered one of the most practical approaches that have been recently studied. ${ }^{4,5}$ In this system, the bulk density of the dosage form is less than that of the gastric fluids, which enables the drug to stay buoyant in the stomach for a longer period time without being influenced by the rate of gastric emptying. The drug is released gradually at the desired rate, and then the residual system is eliminated from the stomach after the drug release. ${ }^{4}$ In an effervescent floating system, hydrophilic polymers are combined with gas-generating agents such as sodium bicarbonate. Sodium bicarbonate is used to liberate $\mathrm{CO}_{2}$ gas upon contact with the gastric fluid that improves the buoyancy of this system while the hydrophilic polymers could control the drug-release rate. ${ }^{6}$

This research accounts for the possible use of swellable hydrophilic polymers as combinations of natural polysaccharides [xanthan gum, glucomannan, tamarind kernel powder (TKP), and chitosan] and semi-synthetic hydrogel [HPMC K15M]. The advantage of these combinations is to get the maximum gel-forming, swelling and remaining buoyant in the gastric fluid of the stomach with a controlled-release rate. Therefore, they are expected to increase the satiety feelings enhancing the weight loss effect of BUP. Researchers have recently interested in the use of biopolymers due to their broad superiority over synthetic polymers. Polysaccharide gums are the most widely used materials because of naturally abundant, biocompatible/degradable, and nonimmunogenic properties. ${ }^{7}$

Apart from successful dosage form preparation and optimization of the novel drug delivery systems with few experiments, design of experiment (DoE) was tailored as an economical approach. This is achieved by optimizing the composition of pharmaceutical formulations using the statistical experimental designs and desirability function as an efficient way to select the optimized formulation. ${ }^{8}$ The optimal mixture designs generally are intended to predict the responses for all suggested formulations of the mixture and to identify the optimal proportions for each of the included factors. The I-optimal mixture design was preferred because it reduces the average variance of prediction over the region of experimentation. I-optimal designs mainly focus on prediction and give better precision in the measurement of the coefficients. ${ }^{9,10}$

The objective of this study is to develop and evaluate swellable gastroretentive floating tablets of BUP using different combinations of natural and synthetic hydrophilic polymers targeting the treatment of comorbid depression with obesity and/or eating disorders. The optimized formula could provide a predictable bioavailability of BUP by prolonging the release and maximizing the swelling index.

\section{Materials and Methods Materials}

Bupropion $\mathrm{HCl}$ and Wellbutrin ${ }^{\circledR} 150 \mathrm{mg}$ sustained-release tablets (reference; Batch no A529170 )(GlaxoSmithKline, Cairo, Egypt), xanthan gum and chitosan (Formurex Pharmaceutical company, California, USA), Konjac glucomannan powder (Hubei Yizhi Konjac Biotechnology Co., Ltd, Yichang, China), Tamarind Kernel Powder (Sciyu Biotech Co., Ltd, Xi'an, China), HPMC K15M, and Sodium bicarbonate (El-Kahera Pharmaceuticals, Cairo, Egypt). Talc, Magnesium stearate, and other reagents and solvents were all pharmaceutical grade and used as received.

\section{Formulation of BUP Floating Tablets Experimental Design}

An I-optimal mixture design using Design Expert $\left.{ }^{(}\right)$software (Version 7, Stat-Ease Inc. Minneapolis, MN, USA) was employed to study the effect of different variables on their corresponding responses. In this design, five independent variables were used to form $280 \mathrm{mg}$ total mixture powders of swelling polymers: Xanthan gum (A), Glucomannan (B), TKP (C), Chitosan (D) and HPMC K15M (E). Lower and upper constraints on the relative amount of each excipient were determined based on the preliminary trials as shown in Table 1. According to the followed I-optimal mixture design and constraints used in this design, 19 different formulations were chosen included four formulations for measuring the lack of fit. Five formulations were performed twice in two separate replicates to estimate the pure experimental error so that a total of 24 runs were generated as shown in Table 2.

\section{Preparation of BUP Floating Tablets}

Twenty-four batches were prepared. Each batch weights 500 grams with a fixed proportion of BUP to mixtures of swellable polymers (150:280 $\mathrm{mg}$ per each tablet). This mixture is then mixed with fixed amounts of Avicel 102 and sodium 
Table I The I-Optimal Mixture Design for Formulation of Swellable Floating Bupropion Tablets Using Mixture of at Least Three Gel-Forming Polymers: Factors and Responses

\begin{tabular}{|c|c|c|c|}
\hline \multirow{2}{*}{$\begin{array}{l}\text { Factors (Independent } \\
\text { Variables) }\end{array}$} & \multicolumn{2}{|c|}{ Levels (mg) } & \multirow{2}{*}{$\begin{array}{l}\text { Total } \\
\text { Mixture }\end{array}$} \\
\hline & Low & High & \\
\hline A: Xanthan Gum & 0 & 120 & $280 \mathrm{mg}$ \\
\hline B: Glucomannan & 0 & 120 & \\
\hline C: TKP & 0 & 120 & \\
\hline D: Chitosan & 0 & 80 & \\
\hline E: HPMC KI5M & 0 & 80 & \\
\hline $\begin{array}{l}\text { Responses (Dependent } \\
\text { Variables) }\end{array}$ & \multicolumn{3}{|c|}{ Desirability Constraints } \\
\hline$Y_{1}:$ Friability (\%) & \multicolumn{3}{|c|}{$0 \% \leq Y_{1} \leq 0.7 \%$, in range } \\
\hline $\mathrm{Y}_{2}: \mathrm{FLT}(\mathrm{sec})$ & \multicolumn{3}{|c|}{ Minimize } \\
\hline $\mathrm{Y}_{3}: \mathrm{Sl}_{4 \mathrm{~h}}(\%)$ & \multicolumn{3}{|c|}{ Maximize } \\
\hline$Y_{4}: S_{8 h}(\%)$ & \multicolumn{3}{|c|}{ Maximize } \\
\hline$Y_{5}:$ Release (\%) after I hour & \multicolumn{3}{|c|}{ Minimize } \\
\hline$Y_{6}:$ Release (\%) after 8 hours & \multicolumn{3}{|c|}{$50 \% \leq Y_{6} \leq 60 \%$, target $=55$} \\
\hline
\end{tabular}

Abbreviations: TKP, tamarind kernel powder; HPMC, hydroxypropyl methylcellulose; FLT, floating lag time; $\mathrm{SI}_{4 h}$, swelling index after 4 hours; $\mathrm{SI}_{8 \mathrm{~h}}$, swelling index after 8 hours.

bicarbonate (50 and $60 \mathrm{mg}$ per each tablet, respectively). Finally, magnesium stearate $(5 \mathrm{mg})$ and purified talc $(5 \mathrm{mg})$ were later added per each tablet. The mixed powders were screened through a 40 -mesh sieve $(425 \mu \mathrm{m})$ and finally compressed into $11 \mathrm{~mm}$ concave tablets each of $550 \mathrm{mg}$ by using a single-punch tablet compression machine under constant pressure to obtain 90 tablets for each formula.

\section{In vitro Evaluation of BUP Floating Tablets Determination of Pre-Compression Parameters}

Bulk density, tapped density, Carr's index, Hausner's ratio, and Angle of repose were done in triplicates for each powder mixture. $^{11}$

\section{Determination of Post-Compression Parameters}

The prepared BUP floating tablets were subjected to weight variation, thickness, diameter, hardness, friability, and uniformity of drug content according to the United States Pharmacopeia (USP) $35 .{ }^{12}$

\section{In vitro Floating Ability}

The test was carried out in triplicate by putting each of the tablets in a USP type-II dissolution apparatus (Copley, USA) with $900 \mathrm{~mL}$ of $0.1 \mathrm{~N} \mathrm{HCl}(\mathrm{pH} \mathrm{1.2)}$. The paddle speed was adjusted at $50 \mathrm{rpm}$, and the temperature was set at $37 \pm 0.5^{\circ} \mathrm{C}$. Their physical state was observed for
$12 \mathrm{~h}$. The in vitro buoyancy was measured for all formulations by investigating floating lag time (FLT) and total floating duration.

\section{In vitro Swelling Study}

The weighed tablets (designated as $\mathrm{W}_{0}$ ) were placed individually in a glass beaker, containing $200 \mathrm{~mL} 0.1 \mathrm{~N} \mathrm{HCl}$ at $37 \pm 0.5^{\circ} \mathrm{C}$. At regular $1 \mathrm{~h}$ time intervals until $8 \mathrm{~h}$, the tablets were removed and reweighed $\left(\mathrm{W}_{\mathrm{t}}\right)$ after removing the excess surface liquid by a filter paper, and then returned back to the beaker. ${ }^{13}$ The swelling Index (SI) was then calculated using the following equation:

$$
\text { Swelling Index } \%(S I)=W_{t}-W_{o} / W_{o} * 100
$$

where $\mathrm{W}_{\mathrm{o}}$ : the initial weight of the tablet; $\mathrm{W}_{\mathrm{t}}$ : the weight of the swollen hydrated tablet at time t. The experiment was performed in triplicates; mean values and standard deviation were calculated.

\section{In vitro Release Study}

The release of BUP from tablets was performed using USP type-II apparatus. The temperature was maintained at $37^{\circ} \mathrm{C}$ with a paddle stirrer at $50 \mathrm{rpm}$. The dissolution medium used was $900 \mathrm{~mL}$ of $0.1 \mathrm{~N} \mathrm{HCl}(\mathrm{pH} \mathrm{1.2)}$. One tablet was kept in the dissolution vessel without much disturbance. At each predetermined time interval $(0,0.5,1,2,3,4,5,6$, 7 , and $8 \mathrm{~h}$ ), a precisely measured sample of the dissolution medium was removed and was replaced by an equal quantity of freshly prepared media. Absorbance of BUP in withdrawn samples was measured at $252 \mathrm{~nm}$ using a UV spectrophotometer. All dissolution runs were performed in triplicates. The results were expressed as the percentage of the cumulative amount of drug as a function of time. ${ }^{14}$

\section{Statistical Analysis of the Mixture Design}

Design-Expert V.7 software was used to develop and evaluate the formulation variables for each response. Six responses were selected; Friability \% $\left(\mathrm{Y}_{1}\right)$, floating lag time $\left(\mathrm{Y}_{2}: \mathrm{FLT}\right)$, Swelling index after 4 hours $\left(\mathrm{Y}_{3}: \mathrm{SI}_{4 \mathrm{~h}}\right)$, Swelling index after 8 hours $\left(\mathrm{Y}_{4}: \mathrm{SI}_{8 \mathrm{~h}}\right)$, as well as Percent of drug released after $1 \mathrm{~h}\left(\mathrm{Y}_{5}\right)$ and $8 \mathrm{~h}\left(\mathrm{Y}_{6}\right)$. Each response was analyzed individually and fitted to linear, quadratic and special cubic models using linear regression.

Analysis of variance (ANOVA) was carried out to estimate the significance of model and term. Probability, p-values, $(p<0.05)$ denoted significance. The statistical parameters used in the evaluation and selection of the best fit model were the p-value of the model, lack-of-fit 
Table 2 A) Experimental Runs, Independent Variables and Their Responses Using I-Optimal Mixture Design of Swellable Floating Bupropion Tablets and B) Predicted and Observed Values for the Six Responses of Optimum Formula

\begin{tabular}{|c|c|c|c|c|c|c|c|c|c|c|c|}
\hline \multicolumn{12}{|l|}{ A) } \\
\hline Formula & A (mg) & B (mg) & C (mg) & D (mg) & E (mg) & $Y_{1}(\%)$ & $\mathbf{Y}_{2}(\mathrm{sec})$ & $Y_{3}(\%)$ & $\mathbf{Y}_{4}(\%)$ & $\mathbf{Y}_{5}(\%)$ & $Y_{6}(\%)$ \\
\hline FTI & 0 & 120 & 120 & 0 & 40 & $0.32 \pm 0.19$ & $24.79 \pm 0.26$ & $262.11 \pm 5.88$ & $196.85 \pm 4.57$ & $74.97 \pm 1.09$ & $100.18 \pm 1.59$ \\
\hline $\mathrm{FT} 2$ & 0 & 120 & 120 & 40 & 0 & $0.49 \pm 0.23$ & $16.63 \pm 0.25$ & $348.06 \pm 4.6$ & $238.04 \pm 5.44$ & $84.475 \pm 1.24$ & $99.81 \pm 3.79$ \\
\hline FT3 & 80 & 60 & 60 & 40 & 40 & $0.85 \pm 0.03$ & $30.43 \pm 0.17$ & $214.62 \pm 3.06$ & $237.72 \pm 3.01$ & $36.24 \pm 2.12$ & $77.67 \pm 2.65$ \\
\hline $\mathrm{FT} 4$ & 80 & 80 & 40 & 40 & 40 & $1.26 \pm 0.3$ & $28.73 \pm 0.19$ & $229.67 \pm 6.53$ & $270.95 \pm 5.51$ & $35.055 \pm 3.08$ & $77.9 \pm 3.15$ \\
\hline FT5 & 80 & 40 & 80 & 40 & 40 & $0.54 \pm 0.02$ & $27.46 \pm 0.19$ & $187.56 \pm 7.8$ & $201.48 \pm 5.62$ & $37.725 \pm 2.98$ & $78.04 \pm 3.2$ \\
\hline FT6 & 80 & 40 & 40 & 60 & 60 & $0.62 \pm 0.3$ & $40.18 \pm 0.16$ & $182.37 \pm 5.46$ & $238.49 \pm 4.31$ & $26.475 \pm 3.66$ & $65.59 \pm 3.43$ \\
\hline FT7 & 80 & 40 & 40 & 40 & 80 & $0.46 \pm 0.12$ & $32.2 \pm 0.78$ & $140.4 \pm 4.27$ & $212.89 \pm 4.17$ & $18.18 \pm 4.26$ & $54.66 \pm 2.81$ \\
\hline FT8 & 80 & 40 & 40 & 80 & 40 & $0.65 \pm 0.25$ & $28.69 \pm 0.54$ & $224.35 \pm 5.71$ & $254.09 \pm 5.15$ & $34.645 \pm 3.88$ & $74.9 \pm 3.01$ \\
\hline FT9 & 80 & 40 & 40 & 80 & 40 & $0.62 \pm 0.18$ & $29.23 \pm 1.16$ & $220 \pm 5.21$ & $251 \pm 4.81$ & $33.995 \pm 4.11$ & $74.5 \pm 3.92$ \\
\hline FIO & 80 & 80 & 80 & 0 & 40 & $0.48 \pm 0.04$ & $60.85 \pm 1.89$ & $206.89 \pm 2.88$ & $212.34 \pm 3.4$ & $42.585 \pm 3.08$ & $90.03 \pm 5.4$ \\
\hline FTII & 80 & 80 & 80 & 0 & 40 & $0.57 \pm 0.24$ & $57.62 \pm 0.4$ & $202 \pm 5.74$ & $219 \pm 4.54$ & $41.15 \pm 2.18$ & $88.3 \pm 3.08$ \\
\hline $\mathrm{FTI2}$ & 80 & 80 & 80 & 40 & 0 & $0.93 \pm 0.28$ & $43.42 \pm 1.53$ & $290.84 \pm 7.95$ & $265.54 \pm 6.3$ & $48.275 \pm 1.61$ & $97.65 \pm 2.07$ \\
\hline $\mathrm{FTI3}$ & 100 & 0 & 100 & 40 & 40 & $0.28 \pm 0.06$ & $28.11 \pm 1.06$ & $133.68 \pm 4.22$ & $153.25 \pm 3.75$ & $31.195 \pm 2.61$ & $78.61 \pm 2.09$ \\
\hline FTI4 & 100 & 100 & 0 & 40 & 40 & $2.93 \pm 0.19$ & $20.13 \pm 2.13$ & $268.95 \pm 5.12$ & $334.43 \pm 4.4 \mathrm{I}$ & $18.245 \pm 2.83$ & $57.64 \pm 2.58$ \\
\hline FTI5 & 100 & 100 & 0 & 40 & 40 & $3.08 \pm 0.15$ & $20.4 \pm 1.06$ & $265 \pm 4.52$ & $337 \pm 4.22$ & $18.705 \pm 3.7$ & $58.01 \pm 2.77$ \\
\hline FTI6 & 100 & 60 & 60 & 0 & 60 & $0.61 \pm 0.36$ & $32.55 \pm 1.32$ & $154.98 \pm 4.06$ & $215.06 \pm 4.42$ & $22.83 \pm 4.79$ & $61.21 \pm 3.15$ \\
\hline FTI7 & 100 & 60 & 60 & 0 & 60 & $0.59 \pm 0.11$ & $33.49 \pm 0.96$ & $156.9 \pm 5.65$ & $217 \pm 4.76$ & $23.1 \pm 3.57$ & $61.4 \pm 3.83$ \\
\hline FT18 & 100 & 60 & 60 & 60 & 0 & $1.41 \pm 0.18$ & $21.02 \pm 1.95$ & $290.41 \pm 3.75$ & $267.85 \pm 3.77$ & $38.425 \pm 4.79$ & $83.02 \pm 3.11$ \\
\hline FTI9 & 120 & 0 & 0 & 80 & 80 & $0.71 \pm 0.04$ & $170 \pm 2.21$ & $130 \pm 3.48$ & $241.52 \pm 3.39$ & $6.92 \pm 2.04$ & $42.53 \pm 4.08$ \\
\hline $\mathrm{FT} 20$ & 120 & 120 & 0 & 0 & 40 & $3.21 \pm 0.04$ & $25.97 \pm 0.49$ & $259.45 \pm 5.57$ & $340.3 \pm 4.92$ & $12.995 \pm 1.63$ & $45.5 \pm 4.4$ \\
\hline FT2I & 120 & 120 & 0 & 40 & 0 & $5.56 \pm 0.6$ & $18.7 \pm 0.47$ & $343.4 \pm 3.72$ & $383.5 \pm 3.69$ & $24.715 \pm 1.35$ & $71.52 \pm 2.5$ \\
\hline $\mathrm{FT} 22$ & 120 & 0 & 120 & 0 & 40 & $0.24 \pm 0.19$ & $55.47 \pm 1.2$ & $97.12 \pm 5.58$ & $125.88 \pm 4.34$ & $28.095 \pm 2.07$ & $79.75 \pm 2.69$ \\
\hline $\mathrm{FT} 23$ & 120 & 0 & 120 & 0 & 40 & $0.23 \pm 0.09$ & $59.57 \pm 1.7$ & $100 \pm 4.9$ & $127 \pm 3.72$ & $27.795 \pm 1.96$ & $80 \pm 2.98$ \\
\hline $\mathrm{FT} 24$ & 120 & 0 & 120 & 40 & 0 & $0.34 \pm 0.12$ & $33.55 \pm 0.47$ & $174.07 \pm 5.07$ & $162.08 \pm 4.58$ & $33.615 \pm 2.72$ & $84.52 \pm 3.75$ \\
\hline \multicolumn{12}{|l|}{ B) } \\
\hline \multicolumn{6}{|c|}{ Predicted values } & 0.7 & 1.595 & 213.589 & 298.178 & 11.897 & 55 \\
\hline \multicolumn{6}{|c|}{ Observed values } & 0.67 & 3.11 & 200.896 & 291.546 & 14.062 & 54.231 \\
\hline \multicolumn{6}{|l|}{ Residual* } & 0.03 & -1.545 & 12.693 & 6.632 & -2.165 & 0.769 \\
\hline
\end{tabular}

Notes: Data are represented as mean \pm SD. *Residual value =predicted value - observed value.

Abbreviations: FT, floating tablet; $A$, xanthan; B, glucomannan; C, tamarind kernel powder (TKP); D, chitosan, E, HPMC KI5M; $Y_{1}$, friability; $Y_{2}$ (FLT), floating lag time; $Y_{3}$ $\left(\mathrm{Sl}_{4 h}\right)$, swelling index after 4 hours; $Y_{4}\left(\mathrm{Sl}_{8 \mathrm{~h}}\right)$, swelling index after 8 hours; $Y_{5}$, percent of drug released after I hour; $Y_{6}$, percent of drug released after 8 hours; SD, standard deviation.

test $\mathrm{p}$-value, coefficient of determination $\left(\mathrm{R}^{2}\right)$, adjusted $\mathrm{R}^{2}$, predicted $\mathrm{R}^{2}$, adequate precision, and predicted residual sum of squares (PRESS). ${ }^{15}$ Also, the graphical presentation of the effects of combined independent variables on simultaneous prediction for each response was presented. Desirability was determined for selecting the optimized formula that was subjected to additional evaluation.

\section{Optimization of the Obtained Data}

The optimization was performed using the statistical program according to the fixed constraints listed in Table 1. The study aims to obtain an optimized floating gastroretentive tablet formula that has a minimum floating lag time and maximum swelling index with modifying the release profile.

The suggested optimized formula was then prepared and assessed in triplicate to verify the validity of the measured optimal formulation factors and predicted responses provided by the software. The difference between both expected and observed responses is known as the residual. The smaller the residual of the optimized batch, the more validity of the design.

\section{Evaluation of the Optimized BUP Tablet Formula}

According to the USP 35, the optimized BUP FT was subjected to post-compression parameters. Also, the kinetics of drug release was analyzed according to zeroorder, first-order, Higuchi, and Korsmeyer-Peppas models. ${ }^{16}$ DDSolver, which is an add-in program for Microsoft Excel was used to model and compare drugrelease profiles. The model with the highest coefficient of determination was considered to be the best fitting one. ${ }^{17}$ 


\section{Differential Scanning Calorimetry (DSC)}

Samples (3-4 mg) of BUP, polymers, and physical form of optimized formula were loaded into an aluminum pan. Powder samples were analyzed by using a differential scanning calorimeter (DSC60 TA-60 WS, Shimadzu, Japan) with a heating rate of $10^{\circ} \mathrm{C} / \mathrm{min}$ over a temperature range of $25-300^{\circ} \mathrm{C}$. The system was purged with nitrogen gas at the rate of $100 \mathrm{~mL} \mathrm{~min}^{-1}$ to maintain an inert atmosphere.

\section{Fourier Transform Infrared Spectroscopy (FTIR)}

To investigate any possible interactions between the drug, polymers and other excipients. FTIR spectra were recorded over a range of $4000450 \mathrm{~cm}^{-1}$ for the drug, excipients, and the physical form of BUP in samples of optimized formula. They were mixed with $500 \mathrm{mg}$ of IR grade potassium bromide powder and then compressed into a disc under pressure.

\section{In vivo Studies}

\section{In vivo Radiographic Study}

To view and track the in vivo gastroretentive behavior of the optimized formulation, in vivo buoyancy study was performed by the incorporation of barium sulfate (radioopaque agent). The amount of the drug $(50 \mathrm{mg})$ was replaced with barium sulfate and other ingredients were kept constant. The study protocol was reviewed, approved (PI 2333-31/12/ 2018), and conducted following the guidelines of the Research Ethics Committee, Faculty of Pharmacy, Cairo University (REC-FOPCU). The study was supervised under the guidance of a radiologist.

Two healthy human volunteers (Males) of $26 \mathrm{yr}$ and $31 \mathrm{yr}$ having heights $160175 \mathrm{~cm}$ weighing $6065 \mathrm{~kg}$ were subjected to abdominal X-ray imaging after giving informed written consent as per declarations of Helsinki (Brazil 2013). The volunteers were given a low-calorie meal after overnight fasting. Half an hour later each subject was administered a barium sulfate-labeled tablet with $200 \mathrm{~mL}$ of water. X-rays were carried out initially and then at various intervals $(0.5,2,5,8,10$ hours) oral post-administration of tablet). During the period of study, volunteers were allowed free access to only water. ${ }^{18}$ The study was fully explained to the volunteers before starting the study, and the informed consent forms were signed by each volunteer as per declarations of Helsinki (Brazil 2013).

\section{Pharmacokinetic Study in Human Volunteers \\ Experimental Design and Sample Collection}

An in vivo study was conducted to compare the pharmacokinetic parameters of BUP from the optimized tablet (test product) and the commercially available, sustainedrelease Wellbutrin ${ }^{\circledR}$ tablets containing $150 \mathrm{mg}$ (reference product), after single oral administration. The protocol and informed consent form were approved by REC-FOPCU (PI 2333). Six male healthy human volunteers were recruited in the study. The study was fully explained to the volunteers before starting the study, and the informed consent forms were signed by each volunteer as per declarations of Helsinki (Brazil 2013).

Two treatments, two periods, randomized, and cross over design was carried out. A 1-week washout period separated the two periods. The volunteers were randomly allocated to one of the two groups of equal size. The volunteers fasted for at least $10 \mathrm{~h}$ with free water access $1 \mathrm{~h}$ before the scheduled time for dosing. Treatments were given with about $240 \mathrm{~mL}$ of water. They were only agreed to take water after $2 \mathrm{~h}$ and a standard meal was given after $4 \mathrm{~h}$ from the administration of treatments.

The study was supervised by a physician who was also responsible for both the safety of the volunteers and samples collection. Samples of venous blood $(5 \mathrm{~mL})$ were collected into heparinized blood tubes via the indwelling cannula immediately prior to oral dosing and at the predetermined time intervals of 1, 2, 3, 4, 5, 6, 8, 24, 48 and $72 \mathrm{~h}$ after dosing. The plasma samples were obtained by centrifuging the samples at $3500 \mathrm{rpm}$ for $10 \mathrm{~min}$, and this was frozen at $-20^{\circ} \mathrm{C}$ in labeled tubes until further analysis.

\section{Sample Preparation}

About $50 \mu \mathrm{L}$ torsemide (from a stock solution of concentration $100 \mathrm{ng} / \mathrm{mL})$ was added to each sample $(0.5 \mathrm{~mL}$ plasma $)$ as an internal standard. BUP and torsemide were extracted using ethyl acetate $(4 \mathrm{~mL})$, and vortexed for $2 \mathrm{~min}$, then centrifuged for $5 \mathrm{~min}$ at $5000 \mathrm{rpm}$ (cooling centrifuge, TGL-20 MB). The supernatant was transferred to other vials filtered through $0.22-1 \mathrm{~m}$ Millipore filter, then evaporated to dryness using vacuum concentrator (Eppendorf Vacufuge plus, Germany).

\section{LC-MS/MS Assay of BUP}

A validated LC-MS/MS method for analyzing plasma BUP concentrations was employed using LC-MS/MS system $\left(\right.$ Shimadzu ${ }^{\circledR}$, Japan) coupled with a triple quadrupole 
detector (API-4500, AB Sciex, Foster, CA, USA). The mobile phase was composed of $80 \%$ acetonitrile, $20 \%$ $0.01 \mathrm{M}$ ammonium formate and $0.1 \%$ formic acid in water. The chromatographic separation was performed on Sunfire C18 column $(4.6 \times 50 \mathrm{~mm}$ i.d., $5 \mu \mathrm{m}$ diameter; Agilent, CA, USA). The injection volume was $10 \mu \mathrm{L}$ and the flow was isocratic with a rate of $1.0 \mathrm{~mL} / \mathrm{min}$. The method has been validated in terms of the selectivity, linearity, precision, accuracy, carryover, extraction recovery, and stability.

\section{Statistical Analysis of the Pharmacokinetic Results}

A non-compartmental pharmacokinetic model was applied to analyze the pharmacokinetic parameters of BUP after the oral administration of two treatments by using the PK solver program. ${ }^{19}$ These parameters included Peak BUP concentration $\left(\mathrm{C}_{\max }, \mathrm{ng} / \mathrm{mL}\right)$ and time to reach $\left(\mathrm{T}_{\max }, \mathrm{h}\right.$.), elimination rate constant $\left(\mathrm{K}_{\mathrm{el}}, \mathrm{h}^{-1}\right)$, terminal half-life $\left(\mathrm{t}_{1 / 2}\right.$, h.), area under the plasma concentration-time curve from time zero to the last observation time point $\left(\mathrm{AUC}_{(0-72)}, \mathrm{ng}\right.$ $\mathrm{h} / \mathrm{mL}$ ) and infinity $\left(\mathrm{AUC}_{(0-\infty)}, \mathrm{ng} \mathrm{h} / \mathrm{mL}\right)$. IBM SPSS Statistics 20 (Armonk, NY, USA) was employed for analysis of all statistical differences in data by using a oneway ANOVA test for the established pharmacokinetic parameters, and P-value $<0.05$ was statistically significant. Nonparametric Kruskal-Wallis test was performed to compare the $T_{\max }$ data obtained from two treatments.

\section{Results and Discussion}

\section{Formulation of BUP Floating Tablets}

The gastroretentive floating tablets were prepared by a single compression technique using combinations of floatable and swellable polymers with sufficient gelling properties, such as xanthan gum, glucomannan, pulverized TKP, chitosan, and HPMC K15M. Magnesium stearate and purified talc were added in the formulations as lubricants. Sodium bicarbonate dissolves in gastric medium and generates gas while Avicel 102 slowly swells to increase tablet volume that helps to mediate floating time together with strong gel-forming polymers. ${ }^{20}$

\section{In vitro Evaluation of BUP Floating Tablets} Determination of Pre-Compression Parameters

Pre-compression parameters for the powders of various formulations showed results within specified limits. ${ }^{21}$ The percentage of Carr's index was found to be in the range of 10.961 to 18.133 and the Hausner ratio was in the range of 1.123 to 1.221 . These values suggest that the powder blends have a range of good to fair flow characteristics and compressibility. Results also were expressed in the range for angle of repose $\left(22.43 \pm 1.48\right.$ to $\left.29.25 \pm 0.71^{\circ} \mathrm{C}\right)$, confirming the good flow property of powders, data not shown.

\section{Determination of Post-Compression Parameters}

All floating tablets were expected to have the same weight and size, but different colors due to the difference in the type and concentration of polymers used in the dosage form. Tablets with high glucomannan concentrations tended to yellow while those with lower glucomannan concentrations were whitish color. Also, tablets with high TKP concentrations tended to brownish color. All tablets were smooth at both sides with a rounded concave shape and no cracks on tablets were observed for any formula.

The mean thickness of BUP FTs ranged from 5.272 to $5.934 \mathrm{~mm}$, while the mean diameter was $11.0 \pm 0.0 \mathrm{~mm}$. Mean hardness lies within the range of $3.25-8.03 \mathrm{~kg} / \mathrm{cm}^{2}$ and it indicates good strength of the floating tablets to withstand physical abrasion. All formulations complied with the pharmacopoeial standard of $\pm 5 \%$ of the labeled claim for weight variation. The percentage of average drug content ranged from $97.03 \%$ to $101.16 \%$ of the labeled claim. ${ }^{14}$

All BUP FTs did not break or show any capping, cracking or chipping during the friability test. All formulations showed acceptable tablet friability results (less than 0.93\%, FT12) except for FT4, FT14, FT15, FT18, FT20 and FT21, whereas, they showed an increase in friability percentage $(>1 \%)$ as shown in Table 2 .

\section{In vitro Floating Ability}

Floating lag time (FLT) indicates the time consumed until the complete floating of the tablet formula on the surface of $0.1 \mathrm{~N} \mathrm{HCl}$ solution. The short FLT and flotation maintained the formulation in the upper GIT by prohibiting them from translocating to the lower GIT, providing sufficient time for a slow drug release from the dosage form. Thus, in vitro floating behavior of floating formulations is crucial to study. The dosage form was considered floated after the tablet completely floated on the test solution.

In this study, sodium bicarbonate was used as a gasforming agent in an optimum concentration of $60 \mathrm{mg}$ to deliver the shortest possible FLT (within a minute) and floating duration of more than 12 hours. So that after the contact with acidic aqueous media, carbon dioxide is generated and entrapped within gelling hydrocolloid, causing density reduction of the system. As the density of the tablet decreases below $1 \mathrm{~g} / \mathrm{mL}$, the tablet becomes buoyant. The 
floating lag time of BUP FTs ranged from $16.63 \pm 0.25 \mathrm{sec}$ for FT2 to $170.00 \pm 2.21 \mathrm{sec}$ for FT19 as shown in Table 2 .

All prepared formulations exhibited good total floating times (more than $12 \mathrm{~h}$ ) due to their contents of polymers with high viscosity that forms strong gel matrices that preserve tablet integrity. This matrix traps gas bubbles to keep the tablets floating for a long time and enhance tablet buoyancy and slow dissolution rates. ${ }^{22}$ Also, none of the formulations disintegrated visually in $0.1 \mathrm{~N} \mathrm{HCl}(\mathrm{pH} 1.2)$ and they remained firm and intact throughout the test. In vitro floating ability of different BUP floating tablets after 12 hours is shown in Figure 1.

The formulations containing the highest concentration of glucomannan had shorter FLT than the formulations containing the highest concentration of TKP with assuming the concentration of other combining polymers was fixed. This can be demonstrated when comparing (FT4 vs FT5), (FT14 and FT15 vs FT13), (FT20 vs FT22 and FT23) and (FT21 vs FT24). It can be explained because formulations containing the highest concentration of glucomannan could swell and form a high viscous matrix structure that was enough strong for gas bubble entrapment when contact with aqueous media and this causes tablet floating. Alternatively, the formulations containing the highest concentration of TKP had a weak gelling and swelling behavior the matrix layer that allowed the gas bubbles responsible for imparting buoyancy to escape. ${ }^{23}$

Chitosan-based formulations had shorter FLT than the corresponding formulations based on HPMC K15M assuming the same concentration of other combining polymers. This can be established when comparing (FT2 vs FT1), (FT8 and FT9 vs FT7), (FT12 vs FT11 and FT10), (FT18 vs FT16 and FT17) and (FT24 vs FT22 and FT23). Chitosan has high swelling capacity, rapid hydration and gel formation at acidic dissolution media $(\mathrm{pH}$ 1.2) as a result of its cationic nature and the amine groups on chitosan are protonated in the acidic environment. ${ }^{24}$ The swelling of chitosan leads to the formation of a dense matrix layer that could efficiently entrap the gas bubbles, resulting in short FLT.

\section{In vitro Swelling Study}

Swelling is an important characteristic of polymer that ensures buoyancy of the matrix tablet and control the drug release. Swelling behavior shows the rate at which the tablet absorbs water from the aqueous media and swells. $^{25}$ Once the hydrophilic polymer matrices contact with the aqueous media, they build up a gel layer around the tablet core that controls the drug release. After forming this gel layer, the hydrophilic polymer matrices were no longer directly exposed to the aqueous solution. Therefore, the degree of swelling dramatically reduced. Meanwhile, this gel served as a buffer zone between the dry region of the tablet and the gastric environment, which also created channels for further water penetration. ${ }^{26}$ As shown in Figure 2, the water uptake of the prepared matrices depends on the type and concentration of polymer used. The swelling mechanisms have been related to the
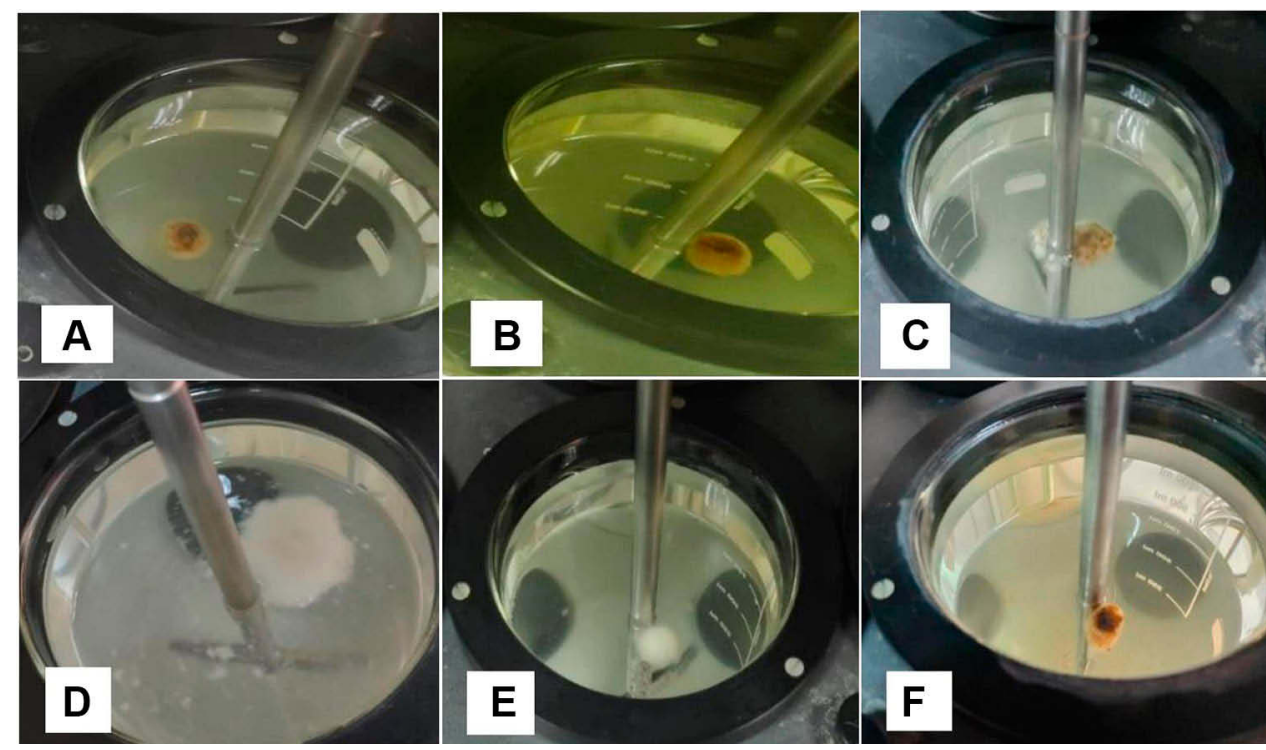

Figure I In vitro floating ability of FT4 (A), FT6 (B), FT9 (C), FTI4 (D), FT20 (E), and FT23 (F) after 12 hours.

Abbreviation: FT, floating tablet. 

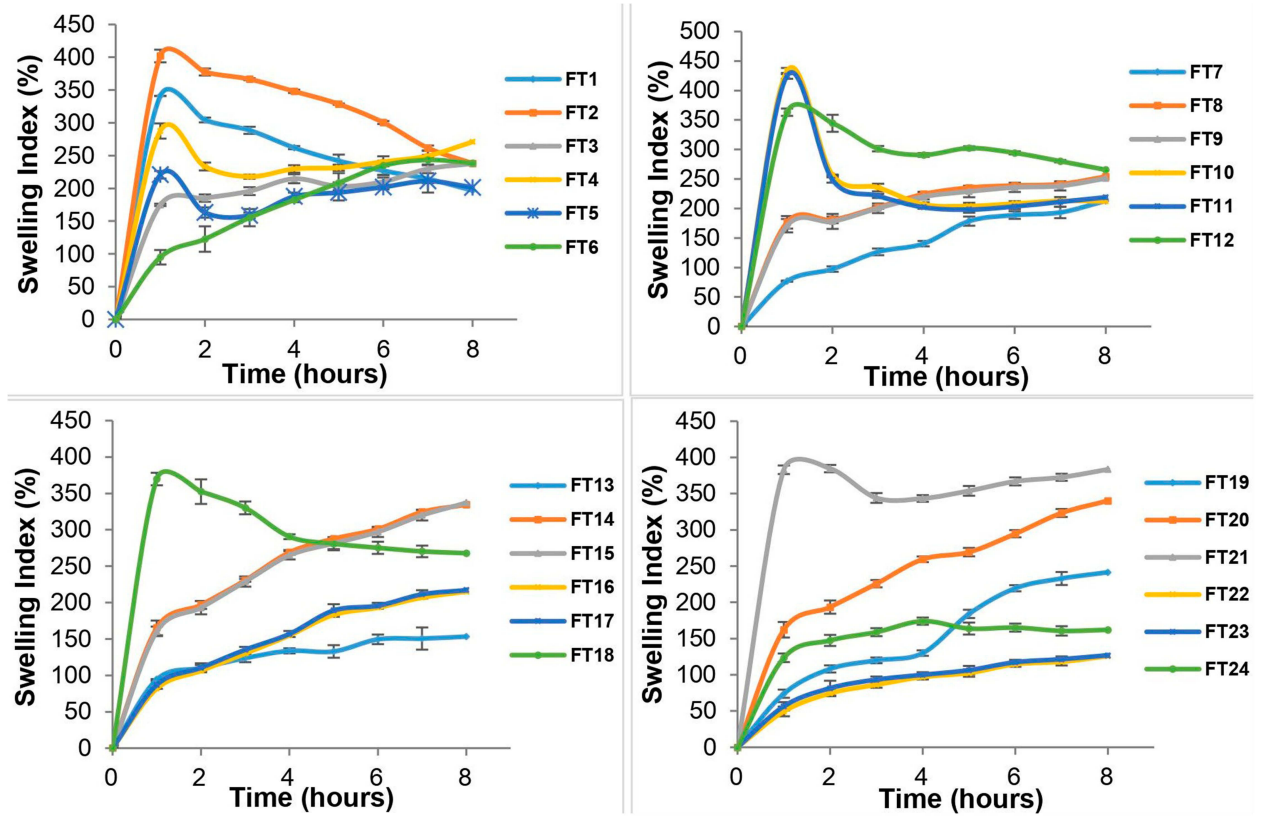

Figure 2 Swelling profiles of all BUP floating tablets with different swellable polymer combinations.

Note: Each point represents the mean values of three tablets $\pm S D$.

Abbreviations: BUP, bupropion; FT, floating tablet; SD, standard deviation.

hydrophilicity of polymers. ${ }^{27}$ The swelling index is used to characterize the hydrophilicity of formulations and the swelling ability of the polymers. The high swelling index, viscosity, and gel-forming behavior of glucomannan were displayed in the previous study. ${ }^{28}$ Also, it is established that the hydrophilic and swellability properties of the polymers affect the $\mathrm{FLT}^{29}$ We can conclude that the swelling and gel-forming properties of glucomannan were stronger than that of TKP polymer. This can be also revealed when comparing (FT4 vs FT5), (FT14 and FT5 vs FT13), (FT20 vs FT22 and FT23) and (FT21 vs FT24). Hence, as the amount of glucomannan was increased, the FLT decreased and swelling index increased, indicating that a high concentration of glucomannan is desirable to achieve minimum FLT and maximum swelling profile.

Swelling index was higher for those tablet formulations containing chitosan than for those containing HPMC $\mathrm{K} 15 \mathrm{M}$. This is due to the highly hydrophilic properties of chitosan as a result of the existence of hydroxyl and amino groups in the structure that have the ability to interact with water molecules. ${ }^{30}$

\section{In vitro Release Study}

The release of drug from the formulation plays an important role in the drug delivery and in determining the therapeutic effect of the medication. An in vitro drug-release study is important to design and test the in vivo activity of drug delivery systems. ${ }^{31}$ In vitro drug-release studies were done to examine the suitability of using different hydrophilic swellable polymers as a matrix for intra-gastric floating drug delivery. Drug release study was conducted in acidic medium $(0.1 \mathrm{~N} \mathrm{HCl})$ since our objective was to formulate a gastroretentive dosage form. The release study was performed in $0.1 \mathrm{~N} \mathrm{HCl}(\mathrm{pH} 1.2)$ at $37 \pm 0.1^{\circ} \mathrm{C}$ at $50 \mathrm{rpm}^{32}$ Release profiles of BUP from different FTs were presented by plotting the percent cumulative amount of drug released in $0.1 \mathrm{~N} \mathrm{HCl}(\mathrm{pH} 1.2)$ against time. The release pattern of a formulation prepared using combinations of natural and synthetic polymers usually generates better results as it follows various mechanisms of release. The effects of different amounts and combinations of polymers on drugrelease properties are presented in Figure 3.

The formulations without xanthan in their composition (FT1 and FT2), were not successful in giving sufficient strength to achieve controlled drug release and reached about $90 \%$ release in $2 \mathrm{~h}$. This finding indicates the gelforming ability and high viscosity of the strong matrix layer formed by xanthan that could lead to a reduction of the effective diffusion coefficient of the drug resulting in a decreased drug release into the dissolution medium. ${ }^{33,34}$

The release pattern of formulations with the highest concentration of glucomannan was slower than the 

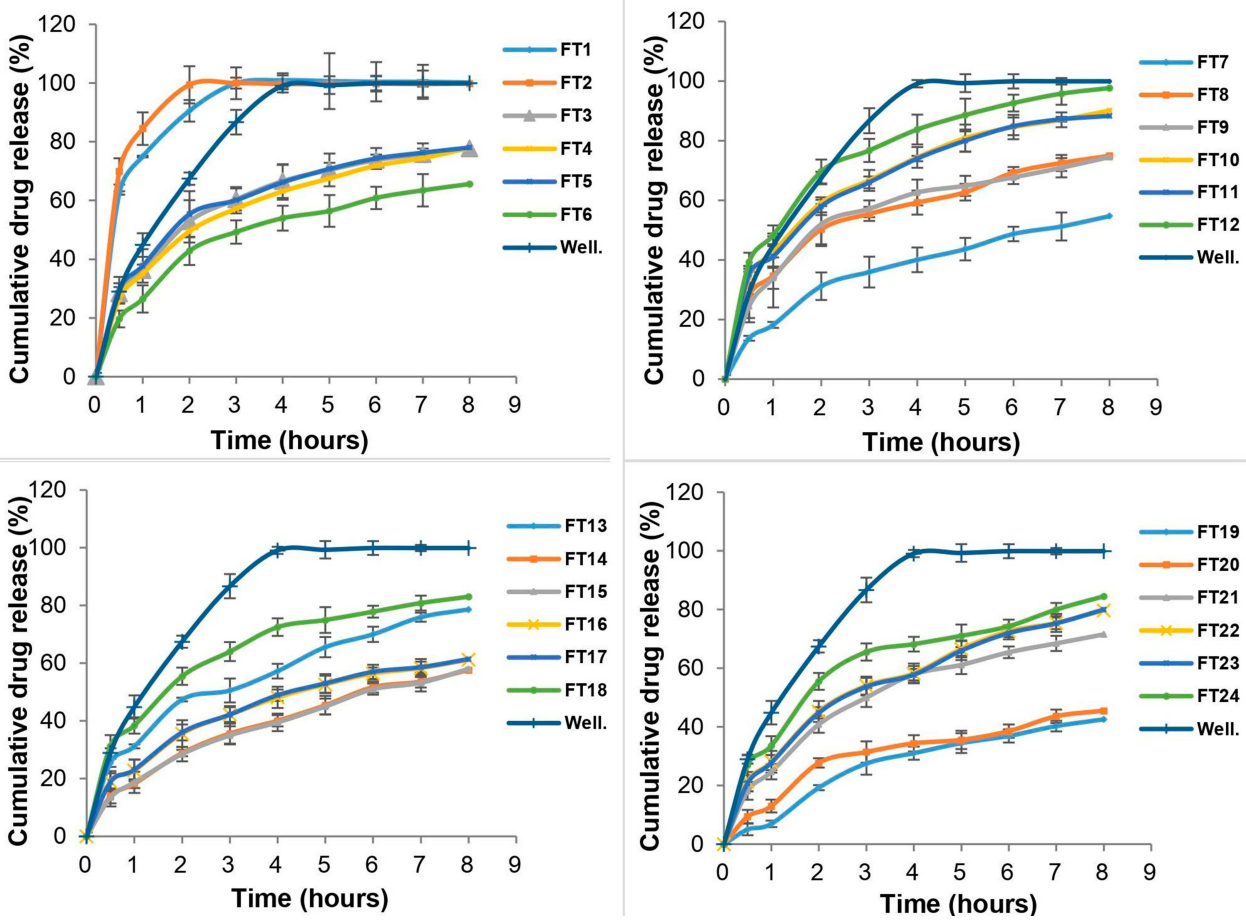

Figure 3 Release profiles of BUP from different swellable floating tablets and commercial Wellbutrin ${ }^{\circledR}$ tablet (Well.).

Note: Each point represents the mean values of three tablets \pm SD.

Abbreviations: BUP, bupropion; FT, floating tablet; Well., Wellbutrin ${ }^{\circledR}$ commercial tablet; SD, standard deviation.

formulations with the highest concentration of TKP in the assumption that the concentration of other combining polymers was fixed. This is owing to the high viscosity and gel-forming ability of glucomannan that formed strong and viscous gel gradually. This gel provides a strong protective matrix for controlling the release process. ${ }^{35}$

It seems that HPMC K15M-based formulations were able to control the release of the drug to a higher extent than that of chitosan-based formulations. This finding could be attributed to the high viscosity and density of HPMC K15M matrix that forms a rigid gel upon contact with aqueous media so that it could control the delivery of the highly water-soluble drug. Moreover, the higher amounts of HPMC show a relatively slower erosion rate of the gelled layer. ${ }^{36}$

\section{Statistical Analysis of the Mixture Design}

I-Optimal Mixture Design has been used as an experimental design to analyze the influence of independent variables on the properties of the drug delivery system. Experimental design was applied for different concentrations of polymers to determine their effect on Friability \% $\left(\mathrm{Y}_{1}\right)$, Floating lag time $\left(\mathrm{Y}_{2}: \mathrm{FLT}\right)$, Swelling index after 4 hours $\left(\mathrm{Y}_{3}: \mathrm{SI}_{4 \mathrm{~h}}\right)$, Swelling index after 8 hours $\left(\mathrm{Y}_{4}: \mathrm{SI}_{8 \mathrm{~h}}\right)$, as well as Percent of drug released after $1 \mathrm{~h}\left(\mathrm{Y}_{5}\right)$ and 8 $\mathrm{h}\left(\mathrm{Y}_{6}\right)$.

\section{Model Fitting and Evaluation for the Responses: $Y_{1}, Y_{2}, Y_{3}, Y_{4}, Y_{5}$, and $Y_{6}$}

The statistical analysis from the Design-Expert ${ }^{\mathbb{R}}$ software indicated the quadratic model for both $\mathrm{Y}_{1}, \mathrm{Y}_{2}, \mathrm{Y}_{5}$, and $\mathrm{Y}_{6}$, and the linear model for $Y_{3}$, and $Y_{4}$ as the best model. The sequential $\mathrm{p}$ values were $<0.0001$ for all responses except $\mathrm{Y}_{5}$ (0.0003), indicating the significance of the model. The lack-of-fit was insignificant for the six models with $\mathrm{p}$ values of $0.8991,0.4985,0.107,0.3203,0.0644$, and 0.3019 for $Y_{1}, Y_{2}, Y_{3}, Y_{4}, Y_{5}$, and $Y_{6}$, respectively. The selected models also showed high $\mathrm{R}^{2}$ values proving strong correlation, good agreement between the adjusted $\mathrm{R}^{2}$ and the predicted $\mathrm{R}^{2}$, and an adequate precision more than 4 indicating high signal-to-noise ratio, ie, adequate signal. ANOVA analysis revealed that linear mixture components have a significant effect on each response with (p-value <0.05). Box-Cox plots were checked for any suggested data transformations. Square root transformation was suggested for $Y_{1}$ and $Y_{2}$, while power transformation was suggested for $Y_{3}$ and $Y_{4}$. No outliers were found for 
any of the six models. Responses $\left(\mathrm{Y}_{5}\right.$ and $\left.\mathrm{Y}_{6}\right)$ had no transformations.

Polynomial equations in terms of coded factors for the measured responses are used as the predictive model for interpretation purposes. The coded equations are shown in equations $(2,3,4,5,6$, and 7$)$.

$$
\begin{aligned}
\sqrt{ } Y_{1}= & +4.61 A+0.46 B+1.25 C-4.88 D-2.85 E \\
& -0.33 A B-9.55 A C+4.36 A D-2.42 A E \\
& -1.51 B C+11.08 B D+3.85 B E+4.21 C D \\
& +4.63 C E+10.27 D E
\end{aligned}
$$

$$
\begin{aligned}
\sqrt{ } Y_{2}= & +6.64 A+9.01 B-5.38 C-2.84 D-14.55 E \\
& +2.26 A B+45.19 A C-0.71 A D+24.94 A E \\
& +42.22 B C-37.35 B D-25.05 B E-41.39 C D \\
& -11.97 C E+149.45 D E
\end{aligned}
$$

$$
\begin{aligned}
\left(Y_{3}\right)^{0.83}= & +60.07 A+189.18 B+61.96 C+149.4 D \\
& -46.08 E \\
\left(Y_{4}\right)^{0.83}= & +112.06 A+170.87 B+4.91 C+127.87 D \\
& +37.50 E
\end{aligned}
$$

$$
\begin{aligned}
Y_{5}= & -3.09 A+113.15 B+11.52 C+78.39 D-152.21 E \\
& -126.4 A B+115.55 A C-78.79 A D+129.38 A E \\
& +127.75 B C-98.05 B D+51.52 B E+32.87 C D \\
& +278.93 C E+282.16 D E
\end{aligned}
$$

$$
\begin{aligned}
Y_{6}= & -136.21 A+302.50 B-47.81 C+94.69 D \\
& -119.55 E-75.60 A B+728.56 A C+326.42 A D \\
& +328.71 A E+5.23 B C-322.63 B D-238.83 B E \\
& -70.57 C D+359.86 C E+339.59 D E
\end{aligned}
$$

The magnitude and sign of the coefficients of the terms in the polynomial equations are used to deduce the effect of their respective terms.

Equation (2) illustrates the effect of the relative proportion of the five studied factors on friability response $\left(\mathrm{Y}_{1}\right)$. The main effect of chitosan (D) provides the largest potent antagonistic on $\mathrm{Y}_{1}$ with a coefficient of $(-4.88)$ as a result of the high binder efficiency of chitosan and its role for matrix formation that previously reported. ${ }^{37}$

Equation (3) illustrates the effect of the relative proportion of the five studied factors on FLT response $\left(\mathrm{Y}_{2}\right)$. The main effect of glucomannan $(\mathrm{B})$ provides the largest potent synergistic influence on $\left(\mathrm{Y}_{2}\right)$ with a coefficient of (+9.01). This could be due to the presence of an abundant hydroxyl group on the saccharide units in the glucomannan structure. As a result, glucomannan could absorb water and show an outstanding property of rapid swelling and high gelling capacity, which is beneficial to the buoyancy ability of formulations. ${ }^{38,39}$

Equation (4) illustrates the effect of the relative proportion of the five studied factors on $\mathrm{SI}_{4 \mathrm{~h}}\left(\mathrm{Y}_{3}\right)$. The main effect of HPMC K15M (E) provides the only antagonistic influence on $\left(\mathrm{Y}_{3}\right)$ with a coefficient of $(-46.08)$ possibly due to the neutral cellulose groups. ${ }^{40}$

Equation (5) illustrates the effect of the relative proportion of the five studied factors on $\mathrm{SI}_{8 \mathrm{~h}}\left(\mathrm{Y}_{4}\right)$. All independent variables (xanthan gum, glucomannan, TKP, chitosan, and HPMC K15M) had significant positive coefficient values $(+112.06,+170.87,+4.91,+127.87$, and +37.50$)$, respectively, on $\left(\mathrm{Y}_{4}\right)$. The main effect of glucomannan provides the largest potent synergistic influence on swelling index percentage at 4 and $8 \mathrm{~h}$ due to the high swelling index, viscosity, and gel-forming ability of glucomannan that previously approved. ${ }^{28,35}$

Equation (6) illustrates the effect of the relative proportion of the five studied factors on the drug release $\%$ after $1 \mathrm{~h}\left(\mathrm{Y}_{5}\right)$. The main effect of HPMC K15M (D) provides the largest significant antagonistic influence with a coefficient of $(-152.21)$. It is explained because HPMC hydrates rapidly on the outer surface of the tablet to form a protective gel layer which is critical to prevent wetting and rapid drug release from the matrix; ${ }^{41}$ however, the release was generally dependent on the other combined polymers. Additionally, the interaction between xanthan and HPMC K15M (AD) had a potent antagonistic effect on $\left(\mathrm{Y}_{5}\right)$ with a coefficient of (-78.79) which is attributed to the intermolecular hydrogen-bonding between them could occur. ${ }^{42}$

Consistently, Equation (7) demonstrates the effect of xanthan gum and HPMC $\mathrm{K} 15 \mathrm{M}$ on the drug release \% after $8 \mathrm{~h}\left(\mathrm{Y}_{6}\right)$ as they had the large significant negative coefficient values ( -136.21 , and -119.55$)$, respectively. This is attributed to the rigid matrix layer of floating tablets containing a higher amount of xanthan and HPMC K15M, in addition to the slow erosion rate of the gel layer from these tablet matrices. ${ }^{34,36}$

\section{Optimization of the Obtained Data}

After applying constraints (Table 1), the Design Expert ${ }^{\circledR}$ v.7 software suggested an optimized formula to be prepared whose overall desirability was 0.782 . The optimum formulation was $73.3 \mathrm{mg}$ xanthan (A), $120 \mathrm{mg}$ glucomannan (B), $8.4 \mathrm{mg}$ tamarind kernel powder (C), $0 \mathrm{mg}$ 
chitosan (D), 78.3 mg HPMC K15M (E). The predicted responses of the suggested formula and corresponding actual experimentally observed values were small demonstrating the validity of the optimization process as shown in Table 2. The contour plot represented in Figure 4 suggesting the optimization of the formulation and the possible predicted values for all responses from the model.

\section{Evaluation of the Optimized BUP Floating Tablet Formula \\ Physical Characterization of Optimized Formula}

The aforementioned procedure resulted in tablets with 11.0 $\pm 0.0 \mathrm{~mm}$ diameter and $5.41 \pm 0.2 \mathrm{~mm}$ thickness. The average weight was $556.1 \pm 2.8 \mathrm{mg}(\mathrm{n}=10)$. Also, the drug content uniformity of the optimized BUP tablet formula was within the acceptance limit $(102.4 \pm 1.5 \%)$ and exhibited an average hardness of $7.8 \pm 0.1 \mathrm{~kg}(\mathrm{n}=6)$. Concerning the release kinetics, criteria for selecting the most appropriate model were based on best fit indicated by the value of the regression coefficient $\left(\mathrm{R}^{2}\right)$ near to 1 . The coefficient $\left(\mathrm{R}^{2}\right)$ of optimized BUP FT indicated that the
Peppas power law equation had the best fit to the experimental data $\left(\mathrm{R}^{2}=0.997\right)$. The value for release exponent (n) was 0.642 , which indicates a non-Fickian diffusion mechanism and that drug release was governed by both diffusion and matrix erosion.

Consequently, these findings confirmed the competence of the I-optimal mixture design to select the optimized formula, which was able to achieve the desired bioavailability and optimum buoyancy with maximum swelling index.

\section{Differential Scanning Calorimetry (DSC)}

DSC is a tool used to examine the melting behavior of materials and to investigate the crystalline or amorphous nature of the drug within the developed formulations. It can be used to identify the melting temperature, heat of fusion, glass transmission and material purity or interaction. The incompatibilities were detected by appearance, shift or disappearance of the corresponding peaks. As demonstrated in Figure 5, the DSC thermogram of pure $\mathrm{BUP}-\mathrm{HCl}$ has one main prominent sharp endothermic melting peak at about $222.83 \mathrm{C}^{\circ}$ (Tm, 213.9

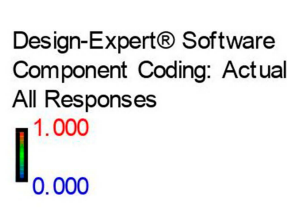

X1 = A: Xanthan $X 2$ = B: Glucomannan $\mathrm{X} 3=\mathrm{C}: \mathrm{TKP}$

Actual Components D: Chitosan $=0$

E: HPMC K = 78.213
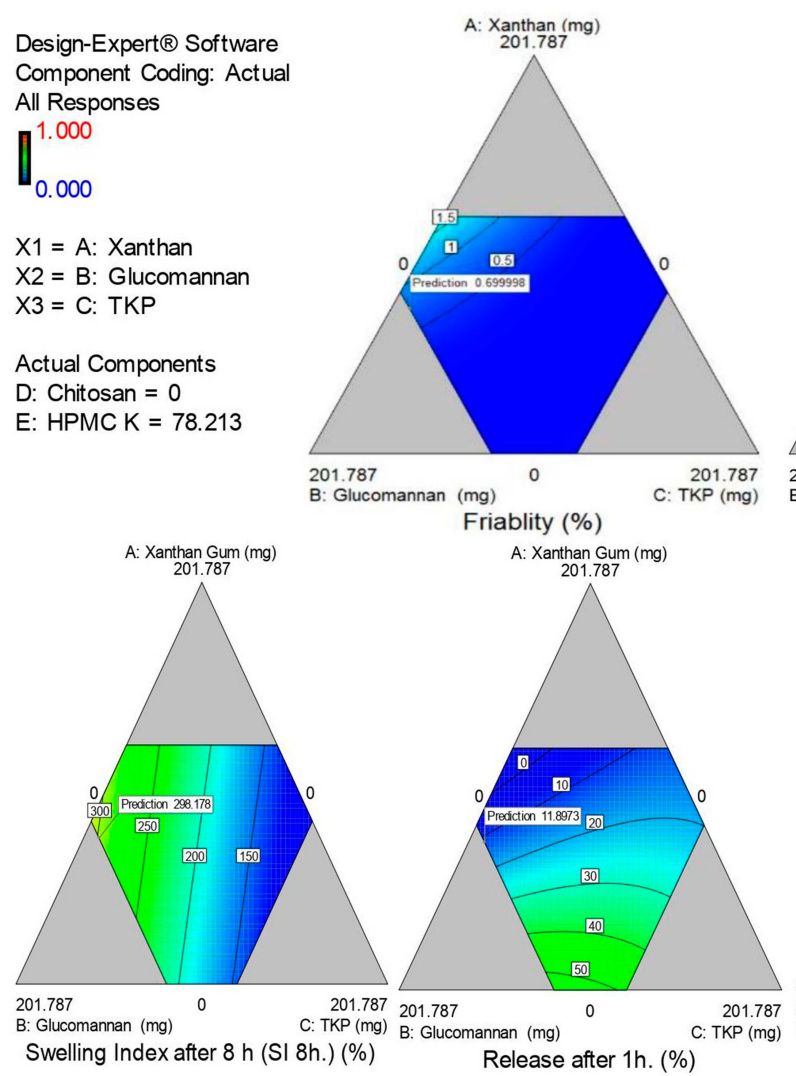
Release after $1 \mathrm{~h} .(\%)$
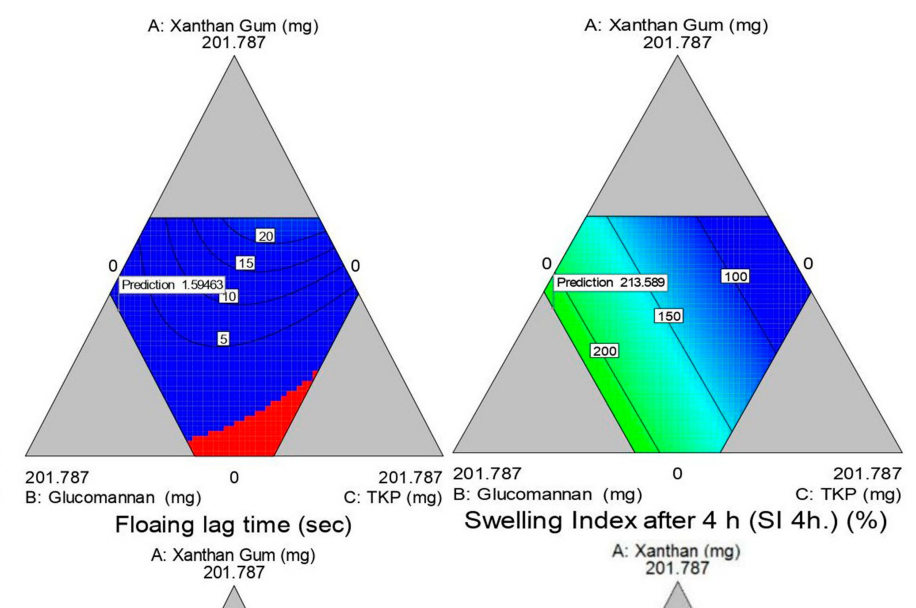

Figure 4 Contour diagram of relationship between three variables, (A) xanthan, (B) glucomannan, and (C) TKP, with actual components of chitosan (D) and HPMC KI5M (E) to simultaneous prediction of all responses.

Abbreviations: TKP, tamarind kernel powder; HPMC, hydroxypropyl methylcellulose. 


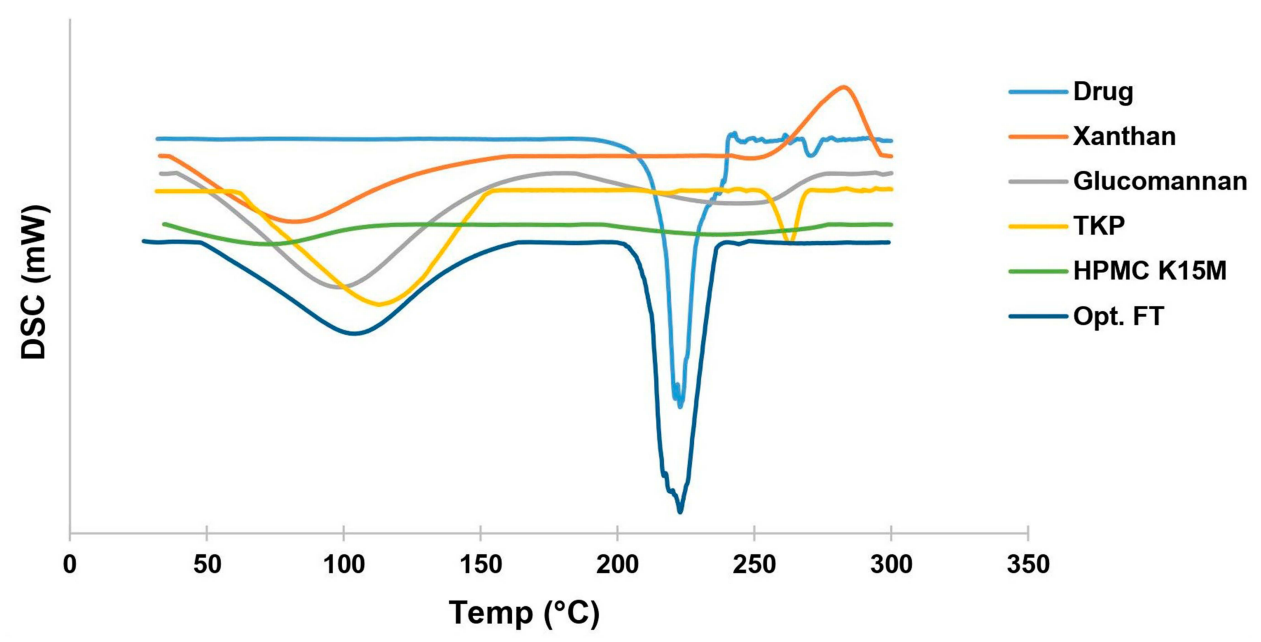

Figure 5 Thermogram DSC of optimized BUP floating tablet (Opt. FT), drug, xanthan, glucomannan, TKP, and HPMC KI5M.

Abbreviations: DSC, differential scanning calorimetry; BUP, bupropion; Opt. FT, optimized bupropion floating tablet; TKP, tamarind kernel powder; HPMC, hydroxypropyl methylcellulose.

onset to $\left.232.2{ }^{\circ} \mathrm{C}\right)$ with melting enthalpy $\left(\Delta \mathrm{H}_{\text {fus }}\right)=$ $326.92,116.8 \mathrm{~J} / \mathrm{g}$ indicating its pure crystalline form.

Thermogram of xanthan gum was characterized by two thermal events: the first endothermic centered at about $81.42^{\circ} \mathrm{C}$, attributed to the loss of water associated with the hydrophilic groups of the polymer, and the second exothermic centered at about $282.7^{\circ} \mathrm{C}$, corresponding to the thermal degradation of xanthan. Meanwhile, broad peaks were observed at $97.84^{\circ} \mathrm{C}$, and $112.84^{\circ} \mathrm{C}$ from the DSC thermogram of pure glucomannan, and TKP, respectively, representing that the melting transition of powders. The melting endotherm of excipients is a broad peak demonstrating an excipient with components of multiple thermal characteristics.

Also, DSC thermograms of HPMC K15M exhibited two broad endothermic peaks; the first peak was at about $72^{\circ} \mathrm{C}$ and the second peak was at about $237.80^{\circ} \mathrm{C}$. It was stated that the thermal analysis of cellulose exhibits an endothermic effect above $100^{\circ} \mathrm{C} .{ }^{43}$

The sharp endothermic peak of BUP was not changed in the thermogram of optimized formula and the drug remained in its intact crystalline form confirming the absence of interaction between the drug and excipients used in the formulation. For further confirmation, the compatibility of the drug with excipients would be investigated by the FTIR.

\section{Fourier Transform Infrared Spectroscopy (FTIR)}

IR spectroscopy was done to investigate the chemical interaction between drug and excipients. The FTIR spectrum of pure Bupropion $\mathrm{HCl}$ showed the peaks at $1693 \mathrm{~cm}^{-1}$ for ketone $\left(\mathrm{C}=\mathrm{O}\right.$ stretch), $1558 \mathrm{~cm}^{-1}$ for amide $(\mathrm{N}-\mathrm{H}$ Out of plane), $1458 \mathrm{~cm}^{-1}$ for aromatic (C-C stretch), $1238 \mathrm{~cm}^{-1}$ for alkyl halides (C-Cl), and $902 \mathrm{~cm}^{-1}$ for dialkyl amine $\left(\mathrm{R}_{2} \mathrm{NH}\right)$.

All excipient spectrums showed a similar wideband observed between $3030-3700 \mathrm{~cm}^{-1}$ that could be attributed to O-H stretching as well as the bands at between 2875 and $2935 \mathrm{~cm}^{-1}$ that could be referred to stretching of aliphatic hydrocarbon $(\mathrm{C}-\mathrm{H})$ groups. The FTIR spectra of xanthan gum showed absorption peaks at 1616.35, 1415.75, $1065 \mathrm{~cm}^{-1}$, whereas for glucomannan, the peaks were detected at 1728.22, 1647.21, 875.68, and $806.25 \mathrm{~cm}^{-1}$. The FTIR spectrum of TKP displayed main peaks at 1662.64, 1415.75, 1076.28, and $1029.99 \mathrm{~cm}^{-1}$. The FTIR spectrum of HPMC displayed characteristic bands at about 1647,1377 , and $1064 \mathrm{~cm}^{-1}$.

The binding fingerprints of BUP-HCl were observed unchanged in the FTIR spectrum of the optimized formula, indicative of no changes in the chemical structure had been occurring during the preparation process (Figure 6).

\section{In vivo Studies \\ Radiographic Study}

In vivo X-ray study was carried out on healthy human volunteers to find the gastric residence time of the optimized FT. The radiographic images revealed that the optimized tablet could remain buoyant for $8-10 \mathrm{~h}$ and its location in the stomach was continuously changed as a function of time, suggesting that the optimized FT did not adhere to the gastric mucosa as shown in Figure 7.

This difference in buoyancy time from in vitro studies may be due to the introduction of a high-density radio-opaque agent 


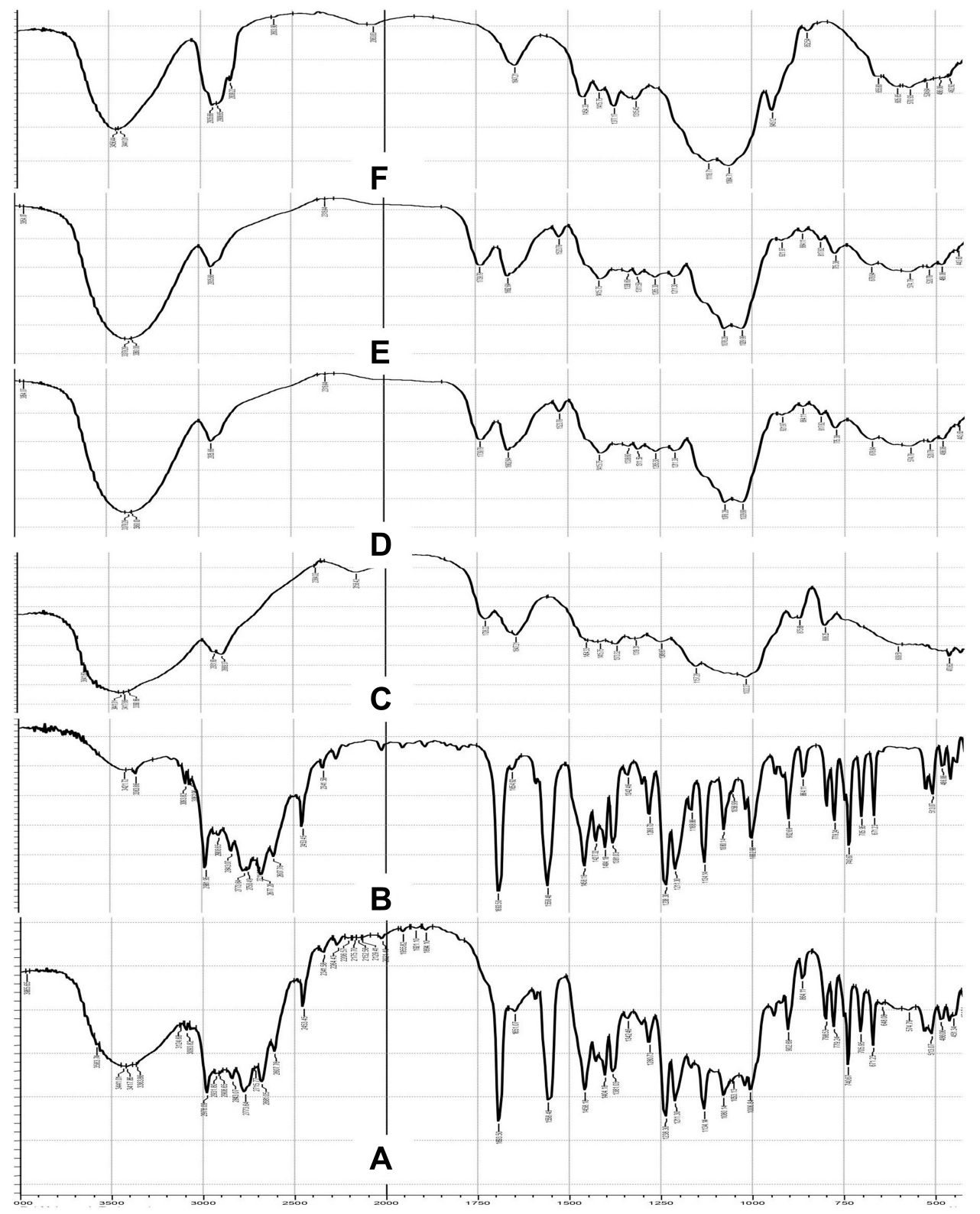

Figure 6 FTIR spectra of optimized BUP floating tablet (a), drug (b), xanthan (c), glucomannan (d), tamarind kernel powder (e), and HPMC KI5M (f). Abbreviations: FTIR, Fourier transform infrared spectroscopy; BUP, bupropion; HPMC, hydroxypropyl methylcellulose.

$\left(4.48 \mathrm{~g} / \mathrm{cm}^{3}\right)$ for radiographic examination and/or the waves of the migratory motor complex in the gastrointestinal tract. Additionally, the migratory myoelectric complex in the stomach may greatly accelerate the migration of floating tablet from the stomach through the small intestine. The migration of the myoelectric complex has been reported to occur in normal individuals about every 2 hours. ${ }^{44}$

\section{Pharmacokinetic Study}

Figure 8 represents the average plasma concentration-time profiles after single oral administration of both marketed Wellbutrin ${ }^{\circledR}$ sustained-release oral tablet and the optimized floating tablet. Different pharmacokinetic parameters were computed by fitting plasma concentration-time profiles to noncompartmental analysis as shown in Table 3.

One-way ANOVA test was performed to compare the data of $\mathrm{C}_{\max }, \mathrm{AUC}_{(0-72)}, \mathrm{AUC}_{(0-\infty)}, \mathrm{K}_{\mathrm{el}}$ and $\mathrm{T}_{1 / 2}$ obtained from two treatments, while nonparametric Kruskal-Wallis test was applied to analyze the $\mathrm{T}_{\max }$ data. Results revealed that the mean peak drug concentration of optimized FT $\mathrm{C}_{\max }(9.116$ $\pm 2.821 \mathrm{ng} / \mathrm{mL}$ ) was comparable with that of the commercial product Wellbutrin ${ }^{\circledR} \mathrm{SR}(10.931 \pm 2.002 \mathrm{ng} / \mathrm{mL})$ and no statistically significant difference $(\mathrm{P}>0.05)$ was detected. Moreover, there was no significant difference $(P>0.05)$ in 

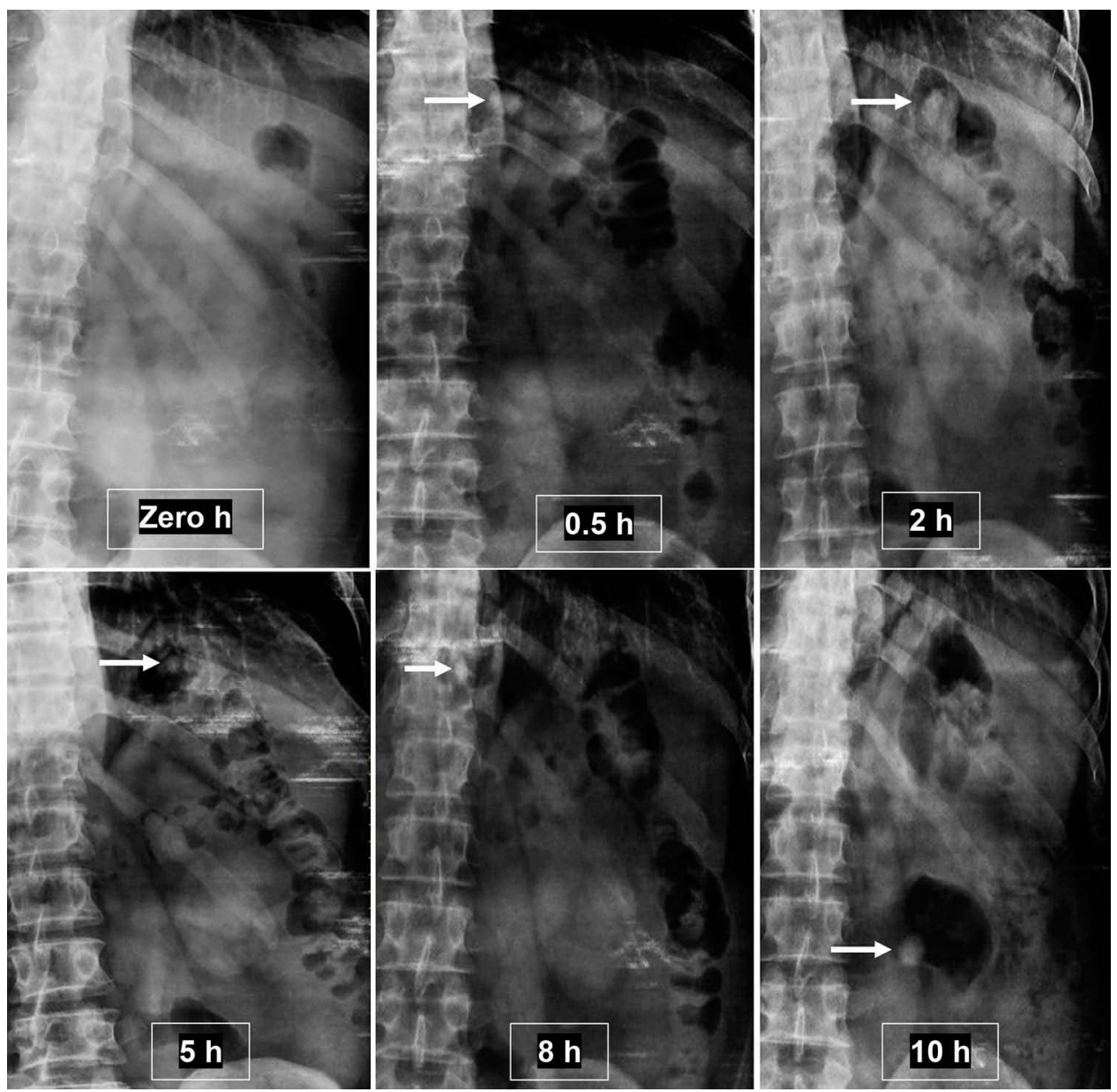

Figure $7 \mathrm{X}$-ray radiographic images indicating the position of optimized floating tablet ( $\mathrm{BaSO}_{4}$-loaded) in the abdomen of a human volunteer at different time intervals (hours).

Note: The location of the tablet is represented with an arrow.

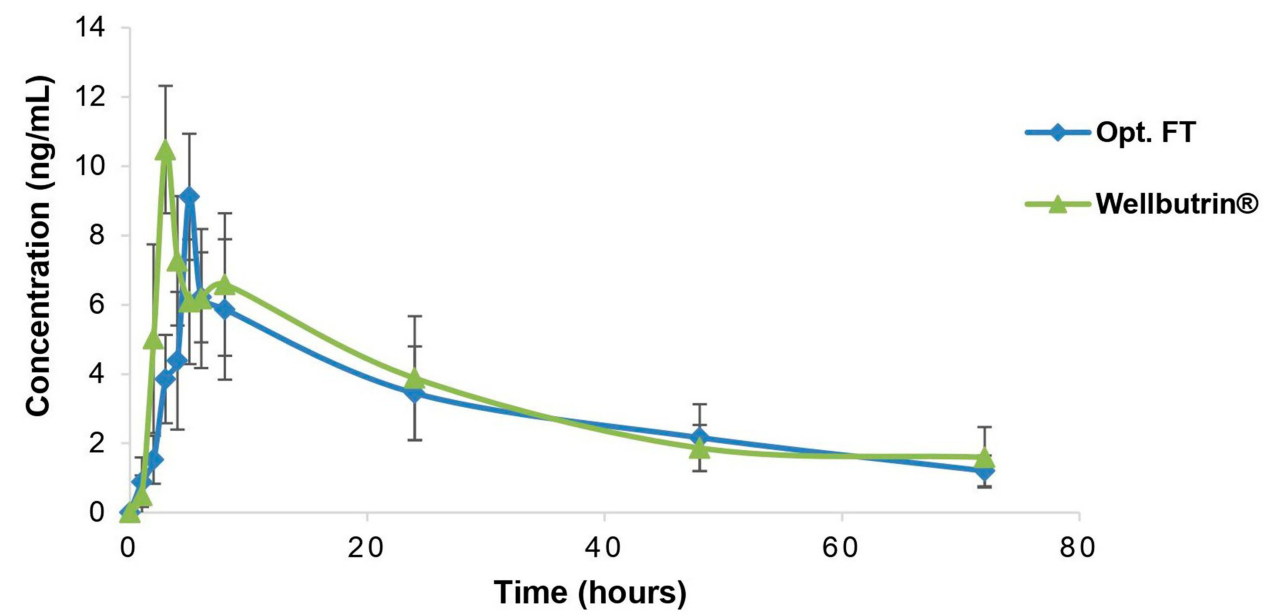

Figure 8 Average plasma concentration-time profiles after single oral administration of both optimized bupropion floating tablets (Opt. FT) and commercial Wellbutrin ${ }^{\circledR}$ SR tablets to human volunteers.

Note: Each point represents mean $\pm S D(n=6)$

Abbreviations: Opt. FT, optimized floating tablet; SR, sustained release; SD, standard deviation. 
Table 3 A) Mean Pharmacokinetic Parameters with ANOVA Analysis of BUP Obtained After Oral Administration of Wellbutrin ${ }^{\circledR}$ SR and Optimized FT to Six Human Volunteers

\begin{tabular}{|l|l|l|l|l|l|l|l|}
\hline A) Mean Pharmacokinetic Parameters \\
\hline
\end{tabular}

Note: Data are presented as mean \pm SD $(n=6)$.

Abbreviations: ANOVA, analysis of variance; BUP, bupropion; SR, sustained-release; FT, floating tablet; $\mathrm{C}_{\max }$, peak plasma concentration; AUC, area under the curve; $\mathrm{K}_{\mathrm{el}}$, elimination rate constant; $T_{\max }$, time to reach maximum peak; h, hours; SD, standard deviation.

the terminal elimination rate constant, $\mathrm{AUC}_{(0-72)}$ value, and $\mathrm{AUC}_{(0-\infty)}$ value between the two formulations.

The mean time to reach the peak concentration $\left(\mathrm{T}_{\max }\right)$ was a statistically significant difference $(\mathrm{p}<0.05)$ among the two treatments. Compared to the marketed product, the relative bioavailability (F) was found to be $96 \%$ referring to $\mathrm{AUC}_{(0-72)}$. Therefore, the optimized floating tablet exhibited the same extent of the commercial tablet with an advantage of the delay in $\mathrm{T}_{\max }$.

\section{Conclusion}

The present research proposed a novel formulation using a combination of natural and synthetic polymers to develop a gastroretentive swellable floating tablet of bupropion. This formulation was optimized to have excellent buoyancy, maximum swelling with a controlled drug-release profile. The optimized tablets had xanthan $(73.3 \mathrm{mg})$, glucomannan $(120 \mathrm{mg})$, tamarind kernel powder (8.4 mg), chitosan (0 mg), HPMC $\mathrm{K} 15 \mathrm{M}(78.3 \mathrm{mg})$ as gel-forming polymers. The in vitro data were validated by in vivo radiographic imaging and in vivo bioavailability study. Thus, the optimized swellable floating tablets of bupropion may be a potential candidate for use in cases of depression comorbid with eating disorders.

\section{Disclosure}

The authors report no conflicts of interest in this work.

\section{References}

1. Plodkowski RA, Nguyen Q, Sundaram U, Nguyen L, Chau DL, St Jeor S. Bupropion and naltrexone: a review of their use individually and in combination for the treatment of obesity. Exp Opinion Pharmacother. 2009;10(6):1069-1081.

2. Layek B, Mandal S. Natural polysaccharides for controlled delivery of oral therapeutics: a recent update. Carbohydr Polym. 2020;230:115617. doi:10.1016/j.carbpol.2019.115617

3. Abd El-Aziz MF, Ismail S, Tadros MI, Elnabarawi MA. Alfuzosin hydrochloride-loaded low-density gastroretentive sponges: development, in vitro characterization and gastroretentive monitoring in healthy volunteers via MRI. Pharm Dev Technol. 2020;1-13.

4. Lin H-L, Chen L-C, Cheng W-T, Cheng W-J, Ho H-O, Sheu M-T. Preparation and characterization of a novel swellable and floating gastroretentive drug delivery system (sfGRDDS) for enhanced oral bioavailability of nilotinib. Pharmaceutics. 2020;12(2):137. doi:10. 3390/pharmaceutics 12020137

5. Choiri S, Sulaiman TS, Rohman A. Assessment of the effect of polymers combination and effervescent component on the drug release of swellable gastro-floating tablet formulation through compartmental modeling-based approach. 2020. Drug Dev Ind Pharm. 1-30. justaccepted. 
6. Rossi A, Conti C, Colombo G, et al. Floating modular drug delivery systems with buoyancy independent of release mechanisms to sustain amoxicillin and clarithromycin intra-gastric concentrations. Drug Dev Ind Pharm. 2016;42(2):332-339. doi:10.3109/03639045.2015. 1054397

7. Jayasree J, Sivaneswari S, Hemalatha G, Preethi N, Mounika B, Murthy SV. Role of various natural, synthetic and semi-synthetic polymers on drug release kinetics of losartan potassium oral controlled release tablets. Int $J$ Pharm Investig. 2014;4(4):183. doi:10.4103/2230-973X.143118

8. Lewis GA, Mathieu D, Phan-Tan-Luu R. Pharmaceutical Experimental Design. New York, USA: CRC press; 1998.

9. Njoroge GG, Simbauni JA, Koske JA. An optimal split plot design for performing a mixture process experiment. Sci J Appl Mathematics Stat. 2017;5:15-23. doi:10.11648/j.sjams.20170501.13

10. Jones B, Goos P. I-optimal versus D-optimal split-plot response surface designs. J Qual Technol. 2012;44(2):85-101. doi:10.1080/ 00224065.2012 .11917886

11. Chappidi SR, Bhargav E, Marikunte V, et al. A cost effective (QbD) approach in the development and optimization of rosiglitazone maleate mucoadhesive extended release tablets in vitro and ex vivo. Adv Pharm Bulletin. 2019;9(2):281. doi:10.15171/apb.2019.032

12. USP.35. Convention USP. United States Pharmacopeia;2011:35

13. Ji J, He X, Yang X-L, et al. The in vitro/vivo evaluation of prepared gastric floating tablets of berberine hydrochloride. AAPS Pharm Sci Tech. 2017;18(6):2149-2156. doi:10.1208/s12249-016-0696-7

14. El Nabarawi MA, Teaima MH, Raa E-M, El Nabarawy NA, Gaber DA. Formulation, release characteristics, and bioavailability study of gastroretentive floating matrix tablet and floating raft system of mebeverine HCl. Drug Des Devel Ther. 2017;11:1081. doi:10.2147/DDDT.S131936

15. Yeom DW, Song YS, Kim SR, et al. Development and optimization of a self-microemulsifying drug delivery system for ator vastatin calcium by using D-optimal mixture design. Int $J$ Nanomedicine. 2015;10:3865.

16. Dash S, Murthy PN, Nath L, Chowdhury P. Kinetic modeling on drug release from controlled drug delivery systems. Acta Pol Pharm. 2010;67(3):217-223.

17. Zhang Y, Huo M, Zhou J, et al. DDSolver: an add-in program for modeling and comparison of drug dissolution profiles. AAPS J. 2010;12(3):263-271. doi:10.1208/s12248-010-9185-1

18. Raza A, Shen N, Li J, Chen Y, Wang J-Y. Formulation of zein based compression coated floating tablets for enhanced gastric retention and tunable drug release. Eur J Pharm Sci. 2019;132:163-173. doi:10. 1016/j.ejps.2019.01.025

19. Zhang Y, Huo M, Zhou J, Xie S. PKSolver: an add-in program for pharmacokinetic and pharmacodynamic data analysis in microsoft excel. Computer Methods Programs Biomed. 2010;99(3):306-314.

20. Razavi M, Karimian H, Yeong CH, et al. Gastroretentive behavior of orally administered radiolabeled tamarind seed formulations in rabbits validated by gamma scintigraphy. Drug Des Devel Ther. 2017;11:1. doi:10.2147/DDDT.S115466

21. Allen L, Ansel HC. Ansel's Pharmaceutical Dosage Forms and Drug Delivery Systems. Lippincott Williams \& Wilkins; 2013.

22. Chen Y-C, Ho H-O, Lee T-Y, Sheu M-T. Physical characterizations and sustained release profiling of gastroretentive drug delivery systems with improved floating and swelling capabilities. Int J Pharm. 2013;441(12):162-169. doi:10.1016/j.ijpharm.2012.12.002

23. Mostafavi A, Emami J, Varshosaz J, Davies NM, Rezazadeh M. Development of a prolonged-release gastroretentive tablet formulation of ciprofloxacin hydrochloride: pharmacokinetic characterization in healthy human volunteers. Int J Pharm. 2011;409(12):128-136. doi:10.1016/j.ijpharm.2011.02.035

24. Sahu A, Verma A, Singh S. Preparation of hydrophilic swelling controlled-release floating matrix tablets containing HPMC and chitosan. Int J Pharm Pharm Sci. 2012;4(1):406-411.
25. Viridén A, Wittgren B, Larsson A. Investigation of critical polymer properties for polymer release and swelling of HPMC matrix tablets. Eur j Pharm Sci. 2009;36(23):297-309. doi:10.1016/j.ejps.2008. 10.021

26. Ai T, Shang L, He C, et al. Development of multi-layered gastric floating tablets based on konjac glucomannan: a modified calcium supplement with enhanced bioavailability. Food Funct. 2019;10 (10):6429-6437. doi:10.1039/C9FO01107B

27. Qi X, Chen H, Rui Y, Yang F, Ma N, Wu Z. Floating tablets for controlled release of ofloxacin via compression coating of hydroxypropyl cellulose combined with effervescent agent. Int J Pharm. 2015;489(12):210-217. doi:10.1016/j.ijpharm.2015.05.007

28. Razavi M, Nyamathulla S, Karimian H, Noordin MI. Novel swellable polymer of orchidaceae family for gastroretentive drug delivery of famotidine. Drug Des Devel Ther. 2014;8:1315. doi:10.2147/DDDT. S68517

29. Rathi M, Medhekar R, Pawar A, Yewale C, Gudsoorkar V. Floating and bioadhesive delivery system of metoprolol succinate: formulation, development and in vitro evaluation. Asian J Pharm. 2014;6:3.

30. Aburahma MH, Mahmoud AA. Biodegradable ocular inserts for sustained delivery of brimonidine tartrate: preparation and in vitro/ in vivo evaluation. Aaps Pharmscitech. 2011;12(4):1335-1347. doi:10.1208/s12249-011-9701-3

31. Esposito E, Menegatti E, Cortesi R. Hyaluronan-based microspheres as tools for drug delivery: a comparative study. Int J Pharm. 2005;288(1):35-49. doi:10.1016/j.ijpharm.2004.09.001

32. Kerdsakundee N, Mahattanadul S, Wiwattanapatapee R. Development and evaluation of gastroretentive raft forming systems incorporating curcumin-Eudragit ${ }^{\circledR}$ EPO solid dispersions for gastric ulcer treatment. Eur J Pharm Biopharm. 2015;94:513-520. doi:10.1016/j.ejpb.2015. 06.024

33. Razavi M, Karimian H, Yeong $\mathrm{CH}$, et al. Gamma scintigraphic study of the hydrodynamically balanced matrix tablets of metformin $\mathrm{HCl}$ in rabbits. Drug Des Devel Ther. 2015;9:3125. doi:10.2147/DDDT. S82935

34. Ali A, Iqbal M, Akhtar N, et al. Assessment of xanthan gum based sustained release matrix tablets containing highly water-soluble propranolol HCl. Acta Pol Pharm. 2013;70(2):283-289.

35. Razavi M, Karimian H, Yeong CH, Chung LY, Nyamathulla S, Noordin MI. Gamma scintigraphic evaluation of floating gastroretentive tablets of metformin $\mathrm{HCl}$ using a combination of three natural polymers in rabbits. Drug Des Devel Ther. 2015;9:4373. doi:10.2147/ DDDT.S86263

36. Jain AK, Söderlind E, Viridén A, et al. The influence of hydroxypropyl methylcellulose (HPMC) molecular weight, concentration and effect of food on in vivo erosion behavior of HPMC matrix tablets. J Controlled Release. 2014;187:50-58. doi:10.1016/j.jconrel.2014.04.058

37. Alsarra IA, El-Bagory I, Bayomi MA. Chitosan and sodium sulfate as excipients in the preparation of prolonged release theophylline tablets. Drug Dev Ind Pharm. 2005;31(45):385-395. doi:10.1080/ 03639040500214563

38. Lopes CM, Bettencourt C, Rossi A, Buttini F, Barata P. Overview on gastroretentive drug delivery systems for improving drug bioavailability. Int J Pharm. 2016;510(1):144-158. doi:10.1016/j.ijpharm.2016.05.016

39. Zhang C, Yang F-Q. Konjac glucomannan, a promising polysaccharide for OCDDS. Carbohydr Polym. 2014;104:175-181. doi:10.1016/ j.carbpol.2013.12.081

40. Yusif RM, Hashim IIA, Mohamed EA, Badria FA-E. Gastrftableoretentive matrix tablets of boswellia oleogum resin: preparation, optimization, in vitro evaluation, and cytoprotective effect on indomethacin-induced gastric ulcer in rabbits. Aaps Pharmscitech. 2016;17(2):328-338. doi:10.1208/s12249-015-0351-8

41. Missaghi S, Fegely KA, Rajabi-Siahboomi AR. Investigation of the effects of hydroalcoholic solutions on textural and rheological properties of various controlled release grades of hypromellose. AAPS PharmSciTech. 2009;10(1):77-80. doi:10.1208/s12249-008-9181-2 
42. Phaechamud T, Darunkaisorn W. Drug release behavior of polymeric matrix filled in capsule. Saudi Pharm J. 2016;24(6):627-634. doi:10.1016/j.jsps.2015.04.003

43. McPhillips H, Craig D, Royall P, Hill V. Characterisation of the glass transition of HPMC using modulated temperature differential scanning calorimetry. Int J Pharm. 1999;180(1):83-90. doi:10.1016/ S0378-5173(98)00407-4
44. Kim JS, Cha KH, Kang SY, et al. In vivo gastric residence and gastroprotective effect of floating gastroretentive tablet of DA-9601, an extract of Artemisia asiatica, in beagle dogs. Drug Des Devel Ther. 2016;10:1917.

\section{Publish your work in this journal}

Drug Design, Development and Therapy is an international, peerreviewed open-access journal that spans the spectrum of drug design and development through to clinical applications. Clinical outcomes, patient safety, and programs for the development and effective, safe, and sustained use of medicines are a feature of the journal, which has also been accepted for indexing on PubMed Central. The manuscript management system is completely online and includes a very quick and fair peer-review system, which is all easy to use. Visit http://www. dovepress.com/testimonials.php to read real quotes from published authors. 